Anthony Érico Guimarães 1

Catarina Macedo Lopes 1

Rubens Pinto de Mello 1

Jeronimo Alencar 1

\title{
Ecologia de mosquitos (Diptera, Culicidae) em áreas do Parque Nacional do Iguaçu, Brasil. 1 - Distribuição por hábitat
}

\author{
Mosquito (Diptera, Culicidae) ecology \\ in the Iguaçu National Park, Brazil. \\ 1 - Habitat distribution
}

${ }^{1}$ Laboratório de Diptera, Departamento de Entomologia, Instituto Oswaldo Cruz. Av. Brasil, 4.365, Rio de Janeiro, $R J$ 21045-900, Brasil. anthony@ioc.fiocruz.br aniratac@ioc.fiocruz.br rmello@ioc.fiocruz.br jalencar@ioc.fiocruz.br

\begin{abstract}
A study of the mosquito fauna in the Iguaçu National Park focused on population behavior in four biotopes with different types of plant cover inside the Park. Systematic bimonthly diurnal and nocturnal human bait and Shannon trap captures were conducted in both forest and domiciliary environments over the course of 24 months. A total of 20,273 adult mosquito specimens belonging to 44 species were collected: Ochlerotatus serratus (10.3\%), Haemagogus leucocelaenus (9.7\%), Mansonia titillans (9.6\%), and Chagasia fajardoi (8.8\%) were the most frequently captured mosquitoes. Anopheles cruzii, Runchomyia theobaldi, Wyeomyia aporonoma, and $\mathrm{Wy}$. confusa were captured almost exclusively in well-preserved areas with dense forest cover. Culex nigripalpus, Oc. pennai, Oc. serratus, Sabethes purpureus, and Sa. albiprivus were captured in three essentially sylvatic biotopes. Species captured in the forest areas around a dam were: An. albitarsis s.l., An. galvaoi, An. evansae, An. fluminensis, Coquillettidia venezuelensis, Cq. juxtamansonia, Wy. quasilongirostris, and Onirion personatum, Ch. fajardoi, Cq. fasciolata, Cq nitens, and Ma. titillans were the most frequently captured species in a residential area.
\end{abstract}

Key words Vector Control; Culicidae; Ecology

Resumo Estabelecemos a influência exercida pela cobertura vegetal de quatro diferentes biótopos em áreas do Parque Nacional do Iguaçu (PNI) sobre a fauna local de mosquitos. Realizaramse capturas bimestrais, em isca humana e armadilha Shannon, em três diferentes períodos do dia, em ambiente silvestre e domiciliar, durante 24 meses consecutivos. Dentre os 20.273 espécimes de fêmeas adultas capturadas, pertencentes a 44 espécies, Ochlerotatus serratus (10,3\%), Haemagogus leucocelaenus (9,7\%), Mansonia titillans (9,6\%) $e$ Chagasia fajardoi $(8,8 \%)$ foram predominantes. Anopheles cruzii, Runchomyia theobaldi, Wyeomyia aporonoma $e$ Wy. confusa ocorreram exclusivamente em áreas com vegetação bem preservada e densa configuração. Culex nigripalpus, Oc. pennai, Oc. serratus, Sabethes purpureus $e$ Sa. albiprivus foram capturados nos três biótopos essencialmente silvestres. Na mata no entorno da represa foram capturadas principalmente An. albitarsis s.l., An. galvaoi, An. evansae, An. fluminensis, Coquillettidia venezuelensis, Cq. juxtamansonia, Wy. quasilongirostris $e$ Onirion personatum. As espécies que apresentaram maiores incidências na área sob ação antrópica foram $\mathrm{Ch}$. fajardoi, Cq. fasciolata, Cq. nitens $e$ Ma. titillans.

Palavras-chave Controle de Vetores; Culicidae; Ecologia 


\section{Introdução}

Incluído desde de 1986 na lista oficial de Patrimônios Naturais da Humanidade pela Organização das Nações Unidas para a Educação, a Ciência e a Cultura (UNESCO), o Parque Nacional do Iguaçu (PNI) é um dos principais centros de convergência do ecoturismo no Brasil. $\mathrm{O}$ intenso fluxo de pessoas às inúmeras atrações do parque torna imperativa a realização de estudos sobre a estrutura biocenótica das populações de mosquitos (Diptera, Culicidae) vetores potenciais de doenças em ambiente silvestre. Além das ações ecoturísticas, são freqüentes intervenções antrópicas tipicamente predatórias: extrativismo vegetal, caça e pesca clandestinas, bem como fixação de posseiros.

Visando a fornecer subsídios ao conhecimento das possíveis implicações da convivência do homem com esses insetos, relatamos os resultados de estudos sobre a incidência da fauna culicideana em quatro diferentes pontos do PNI, no Estado do Paraná, Brasil (Figura 1).

\section{Material e métodos}

Criado em 1939, o PNI ocupa hoje uma área de 185.000 ha com cerca de $400 \mathrm{~km}$ de perímetro, parte em limite direto com rio Iguaçu, e está localizado entre as coordenadas $25^{\circ} 05^{\prime}$ e $25^{\circ} 41^{\prime}$ de latitude sul e $53^{\circ} 40^{\prime}$ e $54^{\circ} 38^{\prime}$ de longitude oeste. $\mathrm{O}$ acesso principal dista cerca de $20 \mathrm{~km}$ do Município de Foz do Iguaçu, a sudoeste do Estado do Paraná, e se estende por outros quatro municípios: Serranópolis do Iguaçu, São Miguel do Iguaçu, Matelândia e Céu Azul.

Além das atrações turísticas: exuberantes quedas d'água, safáris ecológicos pela mata, passeios de barco pelas corredeiras e sobrevôos panorâmicos sobre as cataratas, o PNI foi palco histórico de missões jesuítas para catequese dos índios tupi-guarani. A denominação "Iguaçu" é de origem guarani, significando "água grande" (i = água e guaçu = grande) em referência a opulência do rio e das quedas d'água. Pesquisadores continuam desenvolvendo na região estudos antropológicos e freqüentemente são encontrados importantes sítios arqueológicos.

O clima geral é mesotérmico brando superúmido e sem uma estação tipicamente seca definida. A média pluviométrica anual é de $1.650 \mathrm{~mm}$, com o principal período de chuvas entre outubro e janeiro. Nesse período, são aferidas as temperaturas mais elevadas, podendo atingir $40^{\circ} \mathrm{C}$ em janeiro. Na estação seca, de maio a agosto, ocorrem as temperaturas mais baixas, variando entre $2^{\circ} \mathrm{C}$ e $5^{\circ} \mathrm{C}$ nos dias mais frios. As médias anuais ficam em torno dos 18$20^{\circ} \mathrm{C}$ e conferem à região um clima tipicamente ameno. A umidade relativa do ar é permanente alta e, mesmo no período mais seco, raramente observamos aferições abaixo de $80 \%$.

A vegetação é densa e diversificada. Apesar das características gerais de floresta estacional emidecídua, encontramos extensos fragmentos típicos de savanas, banhados e das matas de araucárias. As principais espécies vegetais são: pau-rosa, alecrim, angico e cedro.

A fauna tem características subtropicais, com influência atlântica direta, e entre os principais representantes encontramos importantes espécies listadas sob risco de extinção: onça pintada, arara azul e papagaio-de-peito-roxo.

Ao longo de 24 meses consecutivos, capturaram-se mosquitos em quatro pontos do PNI, com diferentes configurações ambientais, conforme descritas a seguir:

- Estação A: compreende um dos pontos ao longo dos 12,5km da Estrada do Poço Preto em que a vegetação encontra-se bem preservada e com dois estratos arbóreos definidos. A mata é formada por árvores de grande porte, com espécies pioneiras, e fartamente ornamentadas por epífitas e bromeliáceas, conferindo um aspecto denso à cobertura vegetal (Figura 1). $\mathrm{O}$ solo é recoberto por espessa camada de folhiço e verifica-se uma grande presença de tocas de roedores e marsupiais.

- Estação B: a cerca de $1,5 \mathrm{~km}$, percorridos mata adentro, a partir do quilômetro cinco da estrada secundária que liga a entrada do parque à região das quedas d'água, localiza-se no entorno da represa do rio São João que abastecia a antiga usina hidrelétrica do parque. A vegetação configura-se por três estratos bem definidos, com sub-bosque arbustivo, solos arenosos e férteis (Figura 1). Ao longo do rio São João, que deságua $6 \mathrm{~km}$ abaixo no rio Iguaçu, observamos mata ciliar, remanescente da floresta nativa, com árvores de até $20 \mathrm{~m}$ de altura, e expressiva presença de bromeliáceas.

- Estação C: o ponto de captura encontra-se às margens da mata que circunda a Vila Residencial dos funcionários do parque. A vila compreende dez casas de madeira entre o $2,5 \mathrm{~km}$ da estrada principal e a mata. Embora sob as condições macroclimáticas de toda região do PNI, o local sofre a influência direta da ação antrópica das residências, com vegetação heterogênea entre áreas de plantio e fragmentos nativos (Figura 1).

- Estação D: próximo às principais quedas d'água do parque, o ponto de captura localizase entre a estrada principal e o rio Iguaçu e a 


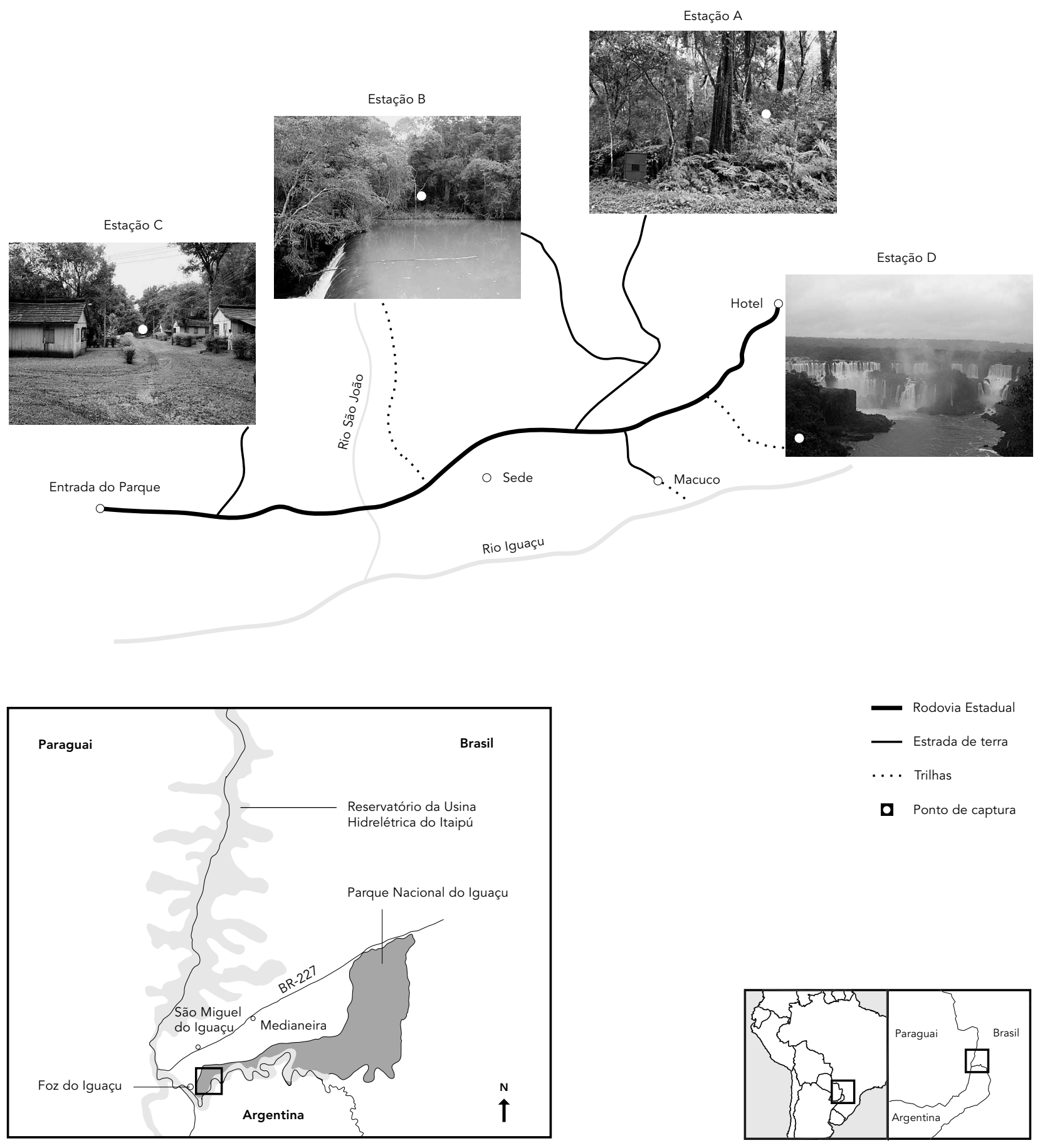


$1 \mathrm{~km}$ da estação de ecoturismo denominada Macuco Safári. A vegetação é formada por mata ciliar de porte médio, árvores finas e retas, com cerca de 15-20 metros de altura, forte influência atlântica e com estrato arbóreo homogêneo (Figura 1).

As capturas foram bimestrais, de janeiro de 1995 a dezembro de 1996, no ambiente silvestre, concomitantemente em isca humana e armadilha luminosa tipo Shannon, com lampião a gás de 100 velas. A armadilha localizava-se a cerca de $150 \mathrm{~m}$ das iscas.

Em três períodos distintos: diurnos (10h às $12 \mathrm{~h}$ e $14 \mathrm{~h}$ às $16 \mathrm{~h}$ ) e noturno ( $18 \mathrm{~h}$ às $21 \mathrm{~h}$ ), dois componentes da equipe do Laboratório de Diptera do Departamento de Entomologia do Instituto Oswaldo Cruz, Fundação Oswaldo Cruz (IOC/FIOCRUZ), com auxílio de tubos de sucção manual, capturavam os mosquitos que neles pousavam para sugar.

Os mosquitos capturados foram mortos pela exposição a clorofórmio e acondicionados em pequenas caixas contendo paraformol, numeradas seqüencialmente com equivalência às fichas ecológicas. Os espécimes foram transportados ao laboratório e fixados em triângulos de papel espetados a alfinetes entomológicos. Cada alfinete recebeu uma etiqueta conferindo-lhe um número e dados de referência. Todos os espécimens foram incorporados à coleção do Departamento de Entomologia do IOC/FIOCRUZ.

A tendência às características ambientais específicas de cada uma das estações de captura foi estabelecida pelo cálculo das médias de Williams $\left(\mathrm{X}_{\mathrm{w}}\right)$. Anopheles albitarsis e An. triannulatus foram considerados como sensu lato.

As abreviaturas genéricas seguiram as recomendações de Reinert (1975). As identificações taxonômicas foram realizadas de acordo com a literatura clássica e as recentes propostas taxonômicas de Harbach \& Peyton (2000) e Judd $(1996,1998)$ para a tribo Sabethini e Reinert (2000) para os gêneros Aedes e Ochlerotatus.

\section{Resultados}

Em amostragens sistematizadas e bimestrais, em quatro diferentes ambientes no PNI, de janeiro de 1995 a dezembro de 1996, foram capturados 20.273 espécimes adultos de 44 espécies de mosquitos (Tabela 1 ).

A subfamília culicinae, sem incluir os sabethini, foi nitidamente a mais abundante com $64 \%$ do total de espécimes capturados, seguida pelos anofelinae com $23 \%$ e da tribo sabethini com $12,6 \%$. As três espécies mais freqüentes em cada grupo seguem esta tendência; culicinae: Ochlerotatus serratus (10,3\%), Haemagogus leucocelaenus (9,7\%) e Mansonia titillans (9,6\%), anophelinae: Chagasia fajardoi (8,8\%), Anopheles albitarsis. (4,5\%) e An. galvaoi (3,0\%), sabethini: Limatus durhami (2,9\%), Trichoprosopon digitatum (1,9\%) e Tr. pallidiventer $(1,6 \%)$ (Tabela 1). A diversidade específica dos três grupos foi muito próxima: culicinae com $38,6 \%$, excluindo os sabethini, que apresentaram $36,4 \%$, e anophelinae, com $25 \%$.

O maior ecletismo foi observado entre os culicíneos com 58,8\% das espécies presentes nos quatro ambientes estudados: 5,9\% em três, $23,5 \%$ em dois e $11,8 \%$ em apenas um deles. Oc. serratus, o mais abundante, ocorreu preferencialmente em ambientes com características silvestres: estação A com $X_{\mathrm{w}}=99,5$, estação B com $X_{\mathrm{w}}=74,6$, estação $\mathrm{D} \operatorname{com} \mathrm{X}_{\mathrm{w}}=60,0$, estação C com $X_{w}=8,0$ (Tabela 2). Oc. scapularis, com igual ecletismo e tendência às estações silvestres, ocorreu principalmente na estação $\mathrm{B}$ $\operatorname{com} X_{w}=69,1$ e $\operatorname{com} X_{W}=16,7$ na $A, X_{w}=12,0$ na $\mathrm{D}$ e $\mathrm{X}_{\mathrm{W}}=4,5$ na C. Oc. pennai ocorreu nas três estações silvestres: $X_{W}=0,7$ na $D, X_{w}=0,4$ na B e $\mathrm{X}_{\mathrm{W}}=0,3$ na A. Oc. terrens foi presente nos ambientes com mata mais densa, respectivamente $\mathrm{X}_{\mathrm{w}}=0,5$ e $\mathrm{X}_{\mathrm{w}}=0,4$ nas estações A e B. Oc. fluviatilis e Oc. fulvus foram exclusivos a um dos ambientes, o primeiro com $X_{\mathrm{w}}=20,1$ na estação D e o segundo com $X_{\mathrm{w}}=1,7$ na B (Tabela 2).

Culex nigripalpus, única espécie do gênero capturada, distribuiu-se de forma semelhante nas amostragens tipicamente silvestres: $\mathrm{X}_{\mathrm{W}}=$ 30,8 na estação $D, X_{w}=25,4$ na $A$ e $X_{w}=23,5$ na B. A presença na estação $C$ foi relativamente tímida, $X_{\mathrm{w}}=4,1$ (Tabela 2).

As três espécies de Psorophora capturadas demonstraram nítida tendência à estação B. Ps. ferox e Ps. albipes, presentes nos quatro ambientes, apresentaram respectivamente $\mathrm{X}_{\mathrm{W}}=$ 99,8 e $\mathrm{X}_{\mathrm{w}}=48,1$ na estação B. Ps. varipes dividiu a preferência entre a estação $\mathrm{B} \operatorname{com} \mathrm{X}_{\mathrm{W}}=1,5 \mathrm{e}$ A com $\mathrm{X}_{\mathrm{W}}=0,8$ (Tabela 2). Hg. leucocelaenus, a terceira espécie mais abundante, foi capturada em todos os ambientes, mas é clara a preferência pelos locais com vegetação densa: $\mathrm{X}_{\mathrm{w}}=$ 103,6 na estação B e $X_{\mathrm{w}}=62,4$ na A (Tabela 2).

Dentre os Mansoniini, observamos que $\mathrm{Co}$ quillettidia nitens, Cq. fasciolata e Mansonia titillans demonstram significativa tendência ao ambiente sob ação antrópica, respectivamente $\mathrm{X}_{\mathrm{W}}=26,9, \mathrm{X}_{\mathrm{w}}=16,5$ e $\mathrm{X}_{\mathrm{w}}=92,7$ na estação C. Cq. chrysonotum e, principalmente, Cq. juxtamansonia, foram capturadas preferencialmente na estação B (Tabela 2).

Chagasia fajardoi foi o único representante dos anophelinae com presença marcante na 
estação C: $\mathrm{X}_{\mathrm{w}}=123,4$. Anopheles lutzi, An. evansae e An. fluminensis ocorreram discretamente nessa estação, respectivamente: $\mathrm{X}_{\mathrm{W}}=$ $1,0, X_{\mathrm{w}}=2,8$ e $X_{\mathrm{w}}=1,5$. As três espécies foram capturadas principalmente na estação $\mathrm{B}: \mathrm{X}_{\mathrm{W}}=$ $5,9, X_{\mathrm{w}}=33,5$ e $\mathrm{X}_{\mathrm{w}}=18,6$. A estação B também foi preferencial para An. albitarsis s.l. $\mathrm{X}_{\mathrm{w}}=$ 107,8, An. galvaoi $\mathrm{X}_{\mathrm{w}}=53,0$ e An. mediopunctatus $\mathrm{X}_{\mathrm{W}}=12,2$. As duas primeiras somente voltariam ocorrer na estação A, respectivamente $\mathrm{X}_{\mathrm{w}}=15,8$ e $\mathrm{X}_{\mathrm{w}}=7,5$. An. mediopunctatus, além dessa estação, $X_{w}=2,1$, também foi encontrado na D: $\mathrm{X}_{\mathrm{W}}=1,1$ (Tabela 2).

Os anofelinos mais presentes na estação A foram: An. cruzii, o único exclusivamente nes- se ambiente, An. triannulatus $\mathrm{X}_{\mathrm{w}}=9,1, A n$. tibiamaculatus $\mathrm{X}_{\mathrm{w}}=6,7$ e An. strodei $\mathrm{X}_{\mathrm{w}}=1,9$. An. triannulatus também foi capturado na estação D: $\mathrm{X}_{\mathrm{W}}=1,1$ e as duas outras na estação $\mathrm{B}$ com respectivamente $\mathrm{X}_{\mathrm{w}}=1,3$ e $\mathrm{X}_{\mathrm{w}}=1,4(\mathrm{Ta}$ bela 2).

Com hábitos tipicamente silvestres, os sabethini foram encontrados com nítida tendência às estações onde a cobertura vegetal é particularmente densa. Algumas espécies ocorreram exclusivamente em uma dessas estações: Runchomyia theobaldi $\mathrm{X}_{\mathrm{w}}=9,8$, Wyeomyia confusa $\mathrm{X}_{\mathrm{w}}=5,0, W y$. aporonoma $\mathrm{X}_{\mathrm{w}}=8,1$ somente na estação A e Sa. identicus $\mathrm{X}_{\mathrm{w}}=1,7, S a$. belisaroi $\mathrm{X}_{\mathrm{W}}=0,7$ na B. Onirion personatum $\mathrm{e}$

Tabela 1

Número absoluto (N) e percentual (\%) dos mosquitos capturados no Parque Nacional do Iguaçu,

Paraná, Brasil, janeiro de 1996 a dezembro de 1997.

\begin{tabular}{|c|c|c|c|}
\hline Espécies & $\% p$ & $\% g$ & $\mathbf{N}$ \\
\hline \multicolumn{4}{|l|}{ Anophelinae } \\
\hline An. (Kerteszia) cruzii Dyar \& Knab, 1908 & 2,5 & 0,6 & 120 \\
\hline An. (Nyssorhynchus) albitarsis Lynch-Arribalzaga, 1878 & 19,3 & 4,5 & 934 \\
\hline An. (Nyssorhynchus) galvaoi Causey, Deane \& Deane, 1943 & 12,9 & 3,0 & 625 \\
\hline An. (Nyssorhynchus) evansae (Brethes, 1926) & 6,1 & 1,4 & 294 \\
\hline An. (Nyssorhynchus) triannulatus (Neiva \& Pinto, 1922) & 2,2 & 0,5 & 107 \\
\hline An. (Nyssorhynchus) tibiamaculatus (Neiva, 1906) & 2,3 & 0,5 & 112 \\
\hline An. (Anopheles) mediopunctatus (Theobald, 1903) & 6,4 & 1,5 & 308 \\
\hline An. (Nyssorhynchus) lutzi Cruz, 1901 & 5,3 & 1,2 & 254 \\
\hline An. (Nyssorhynchus) strodei Root, 1926 & 1,2 & 0,3 & 56 \\
\hline An. (Anopheles) fluminensis Root, 1927 & 4,3 & 1,0 & 209 \\
\hline Ch. fajardoi (Lutz, 1904) & 37,5 & 8,8 & 1.817 \\
\hline Subtotal & 100,0 & 23,3 & 4.836 \\
\hline \multicolumn{4}{|l|}{ Culicinae (exceto Sabethini) } \\
\hline Cx. (Culex) nigripalpus Theobald, 1901 & 6,0 & 3,8 & 795 \\
\hline Cq. (Rhynchotaenia) chrysonotum (Peryassú, 1922) & 6,4 & 4,1 & 853 \\
\hline Cq. (Rhynchotaenia) fasciolata (Arribalzaga, 1891) & 4,9 & 3,1 & 649 \\
\hline Cq. (Rhynchotaenia) nitens (Cerqueira, 1943) & 10,5 & 6,7 & 1.395 \\
\hline Cq. (Rhynchotaenia) venezuelensis (Theobald, 1912) & 1,0 & 0,6 & 132 \\
\hline Cq. (Rhynchotaenia) juxtamansonia (Chagas, 1907) & 1,2 & 0,8 & 163 \\
\hline Ma. (Mansonia) titillans (Walker, 1848) & 15,0 & 9,6 & 2.000 \\
\hline Oc. (Ochlerotatus) pennai (Antunes \& Lane, 1938) & 0,2 & 0,1 & 21 \\
\hline Oc. (Ochlerotatus) serratus (Theobald, 1901) & 16,0 & 10,3 & 2.134 \\
\hline Oc. (Ochlerotatus) scapularis (Rondani, 1848) & 10,0 & 6,4 & 1.333 \\
\hline Oc. (Ochlerotatus) fulvus (Wiedemann, 1828) & 0,3 & 0,2 & 38 \\
\hline Oc. (Ochlerotatus) fluviatilis (Lutz, 1904) & 1,4 & 0,9 & 192 \\
\hline Oc. (Protomacleaya) terrens Walker, 1856 & 0,1 & 0,1 & 19 \\
\hline Ps. (Janthinosoma) ferox (Humboldt, 1820) & 7,0 & 4,5 & 930 \\
\hline Ps. (Janthinosoma) albipes (Theobald, 1907) & 4,5 & 2,9 & 592 \\
\hline Ps. (Janthinosoma) varipes (Coquillett, 1904) & 0,3 & 0,2 & 39 \\
\hline Hg. (Conopostegus) leucocelaenus (Dyar \& Shannon, 1924) & 15,2 & 9,7 & 2.018 \\
\hline Subtotal & 100,0 & 64,0 & 13.303 \\
\hline
\end{tabular}

(continua) 


\begin{tabular}{lrrr} 
Tabela 1 (continuação) & & \\
\hline Espécies & $\% \mathbf{p}$ & \% & N \\
\hline Sabethini & & & 342 \\
Tr. pallidiventer (Lutz, 1905) & 13,0 & 1,6 & 394 \\
Tr. digitatum Rondani, 1848 & 15,0 & 1,9 & 47 \\
Tr. simile Lane \& Cerqueira, 1942 & 1,8 & 0,2 & 79 \\
Ru. (Runchomyia) theobaldi Lane \& Cerqueira, 1942 & 3,0 & 0,4 & 101 \\
Ru. (Runchomyia) reversa Lane \& Cerqueira, 1942 & 3,8 & 0,5 & 193 \\
Wy. mystes (Dyar, 1924) & 7,4 & 0,9 & 79 \\
Wy. aporonoma Dyar \& Knab, 1906 & 3,0 & 0,4 & 40 \\
Wy. (Prosopolepis) confusa (Lutz, 1905) & 1,5 & 0,2 & 177 \\
Wy. (Phoniomyia) quasilongirostris (Theobald, 1907) & 6,7 & 0,9 & 71 \\
On. personatum (Lutz, 1904) & 2,7 & 0,3 & 606 \\
Li. durhami Theobald, 1901 & 23,1 & 2,9 & 156 \\
Sa. (Sabethes) purpureus Theobald, 1907 & 6,0 & 0,8 & 185 \\
Sa. (Sabethes) albiprivus Lutz, 1903 & 7,1 & 0,9 & 7 \\
Sa. (Sabethes) belisaroi Neiva, 1908 & 0,3 & 0,0 & 129 \\
Sa. (Sabethoides) chloropterus (Humbold, 1820) & 4,9 & 0,6 & 18 \\
Sa. (Sabethinus) identicus Dyar \& Knab, 1907 & 0,7 & 0,1 & 2.624 \\
Subtotal & 100,0 & 12,6 & 20.763 \\
Total & 100,0 & 99,9 &
\end{tabular}

Wy. quasilongirostris demonstraram forte tendência para a estação $\mathrm{B}$, respectivamente $\mathrm{X}_{\mathrm{W}}=$ 5,3 e $X_{\mathrm{w}}=25,7$, com tímidas ocorrências na estação D para a primeira $X_{\mathrm{w}}=0,3$, e na estação C para a segunda $\mathrm{X}_{\mathrm{W}}=1,2$. Wy. mystes e Ru. reversa foram capturadas principalmente na estação A: $X_{w}=19,4$ e $X_{w}=5,2$ e na $B: X_{w}=3,8$ e $\mathrm{X}_{\mathrm{w}}=0,8$ (Tabela 2).

Trichoprosopon digitatum, Sabethes purpureus e Sa. albiprivus tiveram distribuição semelhantes. Excluindo a pequena incidência de $T r$. digitatum na estação C: $\mathrm{X}_{\mathrm{W}}=0,4$, as três espécies foram bem representadas nas estações tipicamente silvestres. Enquanto Tr. digitatum demonstrou ligeira tendência pela estação B: $\mathrm{X}_{\mathrm{w}}=16,2$, seguida pela $\mathrm{A} \operatorname{com} \mathrm{X}_{\mathrm{w}}=13,7$; em Sa. Purpureus, a preferência foi pela estação A: $X_{\mathrm{w}}=5,8$, seguida pela B: 2,7 . Sa. albiprivus praticamente não apresentou tendência por uma dessas estações: $\mathrm{X}_{\mathrm{W}}=4,9$ na A e $\mathrm{X}_{\mathrm{w}}=4,8$ na B. As três espécies tiveram a estação D como a terceira maior incidência (Tabela 2 ).

Sa. chloropterus e Tr. simile, embora com tendências ao ambiente silvestre, $\mathrm{X}_{\mathrm{w}}=4,2 \mathrm{e}$ $X_{w}=0,9$ na estação $A ; X_{w}=4,0$ e $X_{w}=1,7$ na $B$, foram proporcionalmente bem representados na estação C: $\mathrm{X}_{\mathrm{w}}=1,4, \mathrm{X}_{\mathrm{w}}=1,3$ e $\mathrm{X}_{\mathrm{w}}=1,9$, respectivamente. Tr. pallidiventer, timidamente presente na estação $\mathrm{C}$ : $\mathrm{X}_{\mathrm{w}}=1,4$, distribuiu-se significativamente nas estações A: $X_{\mathrm{w}}=19,8$, e $\mathrm{B}: \mathrm{X}_{\mathrm{W}}=16,3$. Limatus durhami foi o sabethini com maior incidência na estação $\mathrm{D}$ : $\mathrm{X}_{\mathrm{W}}=44,9$, estando também presente na $\mathrm{A}: \mathrm{X}_{\mathrm{w}}=27,7$, C: $X_{w}=9,8$ e B: $X_{w}=2,7$ (Tabela 2).

\section{Discussão}

Inserido em áreas com cobertura vegetal característica de mata atlântica na costa leste da América do Sul, o PNI apresenta condições ecológicas compatíveis com a ocorrência de espécies de mosquitos típicas de áreas de preservação no Brasil. Entre as particularidades do PNI, constatamos expressiva diversidade de anofelinos em relação a observações em outros parques brasileiros. No PNI, esses mosquitos representaram $23 \%$ das espécies, enquanto em outros estudos essa participação esteve em 14\% no Parque Estadual da Serra do Mar, São Paulo - PESM/SP (Guimarães et al., 2000a, 2000c), $11 \%$ no Parque Nacional da Serra da Bocaina, São Paulo/Rio de Janeiro - PNSB/SPRJ (Guimarães et al., 2000b, 2001); 10\% na Área de Preservação Ambiental Porangaba, Rio de Janeiro - APAPg/RJ (Guimarães et al., 1989), 8\% na Reserva Biológica do Tinguá, Rio de Janeiro - RBT/RJ (Lopes et al., 1999), 5\% no Parque Nacional da Serra dos Órgãos, Rio de Janeiro - PNSO/RJ (Guimarães et al., 1984) e $4 \%$ no Parque Nacional da Chapada dos Guimarães, Mato Grosso - PNCG/MT (dados ainda não publicados). Espécies como An. albitarsis, o anofelino mais abundante no PNI, somente 
Número absoluto $(\mathrm{N})$ e as respectivas médias de Willians $(\mathrm{Xw})$ de mosquitos por estação de captura, em isca humana e armadilha Shannon, no Parque Nacional do Iguaçu, Paraná, Brasil, janeiro de 1995 a dezembro de 1996.

\begin{tabular}{|c|c|c|c|c|c|c|c|c|c|}
\hline \multirow[t]{3}{*}{ Espécies } & \multicolumn{8}{|c|}{ Estações de captura } & \multirow[t]{3}{*}{ Total } \\
\hline & \multicolumn{2}{|c|}{ A } & \multicolumn{2}{|c|}{$B$} & \multicolumn{2}{|c|}{ C } & \multicolumn{2}{|c|}{$D$} & \\
\hline & $X_{w}$ & $\mathrm{~N}$ & $x_{w}$ & $\mathrm{~N}$ & $X_{w}$ & $\mathrm{~N}$ & $X_{w}$ & $\mathrm{~N}$ & \\
\hline An. cruzii & 14,3 & 120 & 0,0 & 0 & 0,0 & 0 & 0,0 & 0 & 120 \\
\hline An. albitarsis s.l. & 15,8 & 138 & 107,8 & 796 & 0,0 & 0 & 0,0 & 0 & 934 \\
\hline An. galvoi & 7,5 & 107 & 53,0 & 518 & 0,0 & 0 & 0,0 & 0 & 625 \\
\hline An. evansae & 0,0 & 0 & 33,5 & 264 & 2,8 & 30 & 0,0 & 0 & 294 \\
\hline An. triannulatus & 9,1 & 90 & 0,0 & 0 & 0,0 & 0 & 1,1 & 17 & 107 \\
\hline An. tibiomaculatus & 6,7 & 71 & 1,3 & 41 & 0,0 & 0 & 0,0 & 0 & 112 \\
\hline An. mediopunctatus & 2,1 & 40 & 12,2 & 248 & 0,0 & 0 & 1,1 & 20 & 308 \\
\hline An. lutzi & 1,6 & 19 & 5,9 & 224 & 1,0 & 11 & 0,0 & 0 & 254 \\
\hline An. strodei & 1,9 & 30 & 1,4 & 26 & 0,0 & 0 & 0,0 & 0 & 56 \\
\hline An. fluminensis & 0,9 & 13 & 18,6 & 177 & 1,5 & 19 & 0,0 & 0 & 209 \\
\hline Ch. fajardoi & 69,1 & 468 & 35,4 & 445 & 123,4 & 817 & 7,0 & 87 & 1.817 \\
\hline Cx. nigripalpus & 25,4 & 205 & 23,5 & 250 & 4,1 & 30 & 30,8 & 310 & 795 \\
\hline Cq. crysonotum & 24,4 & 250 & 25,9 & 473 & 5,5 & 51 & 3,7 & 79 & 853 \\
\hline Cq. fasciolata & 8,6 & 360 & 4,6 & 178 & 16,5 & 108 & 0,3 & 3 & 649 \\
\hline Cq. nitens & 15,9 & 271 & 10,0 & 635 & 26,9 & 474 & 0,6 & 15 & 1.395 \\
\hline Cq. venezuelensis & 0,0 & 0 & 15,4 & 115 & 1,4 & 17 & 0,0 & 0 & 132 \\
\hline Cq. juxtamansonia & 0,0 & 0 & 12,0 & 160 & 0,3 & 3 & 0,0 & 0 & 163 \\
\hline Ma. titillans & 21,3 & 235 & 74,4 & 880 & 92,7 & 879 & 0,4 & 6 & 2.000 \\
\hline Oc. pennai & 0,3 & 5 & 0,4 & 7 & 0,0 & 0 & 0,7 & 9 & 21 \\
\hline Oc. serratus & 99,5 & 879 & 74,6 & 641 & 8,0 & 70 & 60,0 & 544 & 2.134 \\
\hline Oc. scapularis & 16,7 & 346 & 69,1 & 762 & 4,5 & 46 & 12,0 & 179 & 1.333 \\
\hline Oc. fulvus & 0,0 & 0 & 1,7 & 38 & 0,0 & 0 & 0,0 & 0 & 38 \\
\hline Oc. fluviatilis & 0,0 & 0 & 0,0 & 0 & 0,0 & 0 & 20,1 & 192 & 192 \\
\hline Oc. terrens & 0,5 & 12 & 0,4 & 7 & 0,0 & 0 & 0,0 & 0 & 19 \\
\hline Ps. ferox & 10,2 & 93 & 99,8 & 717 & 1,0 & 14 & 8,7 & 106 & 930 \\
\hline Ps. albipes & 2,3 & 68 & 48,1 & 428 & 2,2 & 28 & 2,1 & 68 & 592 \\
\hline Ps. varipes & 0,8 & 9 & 1,5 & 30 & 0,0 & 0 & 0,0 & 0 & 39 \\
\hline Hg. leucocelaenus & 62,4 & 911 & 103,6 & 910 & 10,4 & 139 & 7,9 & 58 & 2.018 \\
\hline Tr. pallidiventer & 19,8 & 124 & 16,3 & 201 & 1,4 & 17 & 0,0 & 0 & 342 \\
\hline Tr. digitatum & 13,7 & 227 & 16,2 & 124 & 0,4 & 6 & 4,6 & 37 & 394 \\
\hline Tr. simile & 0,9 & 13 & 1,7 & 23 & 1,2 & 11 & 0,0 & 0 & 47 \\
\hline Ru. theobaldi & 9,8 & 79 & 0,0 & 0 & 0,0 & 0 & 0,0 & 0 & 79 \\
\hline Ru. reversa & 5,2 & 72 & 0,8 & 29 & 0,0 & 0 & 0,0 & 0 & 101 \\
\hline Wy. mystes & 19,4 & 152 & 3,8 & 41 & 0,0 & 0 & 0,0 & 0 & 193 \\
\hline Wy. aporonoma & 8,1 & 79 & 0,0 & 0 & 0,0 & 0 & 0,0 & 0 & 79 \\
\hline Wy. confusa & 5,0 & 40 & 0,0 & 0 & 0,0 & 0 & 0,0 & 0 & 40 \\
\hline Wy. quasilongirostris & 0,0 & 0 & 25,7 & 165 & 1,2 & 12 & 0,0 & 0 & 177 \\
\hline On. personatum & 0,0 & 0 & 5,3 & 66 & 0,0 & 0 & 0,3 & 5 & 71 \\
\hline Li. durhami & 27,7 & 209 & 2,7 & 44 & 9,8 & 69 & 44,9 & 284 & 606 \\
\hline Sa. purpureus & 5,8 & 73 & 2,7 & 45 & 0,0 & 0 & 2,5 & 38 & 156 \\
\hline Sa. albiprivus & 4,9 & 76 & 4,8 & 75 & 0,0 & 0 & 2,1 & 34 & 185 \\
\hline Sa. belisaroi & 0,0 & 0 & 0,7 & 7 & 0,0 & 0 & 0,0 & 0 & 7 \\
\hline Sa. chloropterus & 4,2 & 53 & 4,0 & 59 & 1,9 & 17 & 0,0 & 0 & 129 \\
\hline Sa. identicus & 0,0 & 0 & 1,7 & 18 & 0,0 & 0 & 0,0 & 0 & 18 \\
\hline Total & & 5.937 & & 9.867 & & 2.868 & & 2.091 & 20.763 \\
\hline
\end{tabular}


haviam sido encontradas no PESM e com valores em $\mathrm{X}_{\mathrm{W}}$ que não expressaram significância na preferência por determinado ambiente (Guimarães et al., 2000a). Apesar do relativo ecletismo quanto ao tipo de criadouro, as expressivas ocorrências do An. albitarsis, An. galvaoi, An. evansae, An. mediopunctatus, An. lutzi e An. fluminensis, na estação B, local da represa do rio São João, e do An. strodei, An. tibiamaculatus e An. triannulatus, na estação A, área de mata densa e com alagados, devem estar diretamente relacionadas ao favorecimento desses ambientes ao desenvolvimento das formas imaturas desses anofelinos (Figura $1 \mathrm{e}$ Tabela 2).

Realizando estudos em áreas alagadas pelo reservatório da Usina Hidrelétrica de Itaipu, também no sudoeste do Estado do Paraná, Guimarães et al. (1997) assinalam a ocorrência do An. albitarsis, An. galvaoi, An. evansae e An. triannulatus e comentam a possível relação entre a presença do An. albitarsis e os casos de malária ocorridos na região entre 1989/1992. Forattini et al. (1995a), trabalhando em áreas alagadas para cultivo de arroz no estado de São Paulo, também ressaltam a presença desses anofelinos nesses criadouros.

As amostragens em áreas de mata próximas aos domicílios na estação C demonstraram que a fauna anofélica no PNI comportase como tipicamente silvestre. Espécies que em outras oportunidades se mostraram passíveis de adaptação ao convívio humano não repetiram essas tendências no PNI. An. cruzii, capturado por Guimarães et al. (2000a, 2000b), em domicílios localizados respectivamente no PESM e PNSB, apresentou, no PNI, o caráter assinantrópico observado por Forattini et al. (1993a, 1993b), que condicionam a sua presença no domicílio exclusivamente para hematofagia e em condições excepcionais, provocadas pela ocasional invasão do homem ao meio silvestre. No PNI, ao contrário do que ocorre no PESM e PNSB, os domicílios estão localizados na periferia do parque (Figura 1) e as incidências de anofelinos nessas amostragens estiveram restritas à escassa presença do An. evansae, An. fluminensis e An. lutzi (Tabela 2) - dois últimos também presentes no PNSB e $A n$. fluminensis no PESM.

Ch. fajardoi foi o único anophelinae com preferência pela área no entorno dos domicílios na estação C. Essa tendência, também observada em alguns culicinae: Cq. fasciolata, Cq. nitens e Ma. titillans, confirma observações anteriores que associam esse comportamento a atração por fontes luminosas em áreas de mata degradadas pela ação do homem (Forattini et al., 1981; Guimarães et al., 1989, 2000a, 2000b).

O represamento do Rio São João e a exuberante vegetação ciliar no seu entorno (Figura 1) pareceram favorecer as incidências na estação B. Entre os mosquitos encontrados no PNI, $86,4 \%$ estiveram presentes nessa estação e $52,3 \%$ foram aí capturados preferencialmente (Tabela 2).

Em diferentes áreas de mata atlântica na região Sudeste, têm sido encontradas muitas das espécies de mosquitos capturadas no PNI: $C q$. crysonotum, Cq. venezuelensis, Cq. juxtamansonia, Oc. scapularis, Oc. fulvus, Hg. leucocelaenus, Ps. ferox, Ps. albipes, Ps. varipes, Tr. digitatum, Tr. simile, Wy. quasilongirostris, On. personatum, Sa. identicus e Sa. belisaroi (Forattini et al., 1978a, 1981, 1986a, 1986b, 1993b; Guimarães \& Arlé, 1984; Guimarães et al., 1989, 2000a, 2000b). Ao compararmos áreas com cobertura vegetal semelhantes, com (estação B) ou sem a influência direta de grandes coleções d'água (estação A), observamos a tendência dessas mesmas espécies pela primeira. Outras tantas, embora mantenham as maiores incidências em áreas típicas de mata densa e fechada (estação A), também parecem ser influenciadas com as possibilidades oferecidas pela proximidade permanente da represa na estação B: Oc. serratus, Oc. terrens, Tr. pallidiventer, Ru. reversa, Wy. mystes, Sa. chloropterus, Sa. purpureus e Sa. albiprivus (Figura 1 e Tabela 2).

Apesar de os dados demonstrarem que as condições encontradas na estação B favoreceram a maioria das espécies, muitas delas não apresentam relatos na literatura que as relacione com a utilização de grandes coleções d'água como criadouros. Essa marcante ocorrência, inclusive de sabethini, deve-se principalmente à presença de árvores ornamentadas com bromeliáceas no entorno da represa e a produção de frutos que, uma vez no solo, transformam-se em criadouros potenciais. Devemos considerar ainda que o cenário global da estação B favorece a presença de mamíferos e aves que certamente são fontes de alimentação para muitos desses mosquitos.

Diferente do observado em outras áreas de preservação ambiental (Guimarães et al., 1984, 1989, 2000a, 2000b; Lopes et al., 1999), Oc. fluviatilis teve incidência no PNI restrita à estação D. Certamente, a potencialidade dos criadouros nas várias aflorações de rocha existentes, pela proximidade entre o ponto de captura e o Rio Iguaçu (Figura 1), colaboraram nessas ocorrências. A mesma possibilidade podemos pressupor para as incidências preferenciais observadas para Oc. pennai e Li. durhami. Esse último vem apresentando, em várias oportuni- 
dades, grande capacidade de adaptação a diferentes condições que lhe são impostas. Exceto pela baixa incidência na estação B, $L i$. durhami ocorreu nas estações A e D de acordo com as observações de Forattini et al. (1978b; 1993b) e Guimarães et al. (1989; 2000a), que capturaram a espécie exclusivamente em ambiente silvestre; sendo também encontrado na estação C, demonstrando a possibilidade de domiciliação observada por Guimarães \& Arlé (1984).

Vários são os relatos sobre a presença do $O c$. scapularis em áreas silvestres sob intensa ação antrópica (Forattini et al., 1978a, 1978b, 1981, 1993c, 1995a; Guimarães et al., 1989, 2000a). Entretanto, embora nos pareça que a espécie se encontre em claro processo de adaptação ao convívio humano, no PNI foi discreta a sua incidência sob tais condições na estação C. $O c$. serratus ocorreu em acordo com as observações anteriores de Forattini et al. (1978a, 1978b, 1981, 1986a, 1995a) Guimarães et al. (2000a) e Lopes et al. (1999), que a consideram como preferencialmente silvestre. Forattini et al. (1995b) observam que, após terem sido realizadas intensas modificações ambientais em áreas do Vale da Ribeira, São Paulo, o Oc. scapularis teve um aumento populacional de 16 vezes e $O c$. serratus um declínio de 284 vezes.

Condições típicas de mata densa e a presença de criadouros específicos, bromeliáceas e ocos de árvores, por exemplo, podem ter sido responsáveis pelas incidências de alguns culicídeos na estação A. Além do An. cruzii, comentado anteriormente, $R u$. theobaldi, $W y$. aporonoma e Wy. confusa foram encontradas exclusivamente nessas condições. A ocorrência dos três sabethini esteve de acordo com os relatos de Forattini et al. (1986a, 1993b) e Guimarães et al. (1989, 2000a) e para o primeiro em Guimarães et al. (2000b).

Sa. identicus e Sa. belisaroi foram capturados exclusivamente na estação B, Guimarães \& Arlé (1984) e Guimarães et al. (1989) relatam observações semelhantes em relação ao primeiro.

Algumas espécies demonstraram certo ecletismo para pelo menos três das possibilidades de ocorrência estabelecidas no PNI. Cx. nigripalpus, Sa. purpureus e Sa. albiprivus, distribuíram-se pelas áreas com características silvestres, estações A, B e D. Embora a tendência pelo ambiente silvestre do Cx. nigripalpus esteja em acordo com as observações de Guimarães et al. (2000a), a sua incidência na estação C reforça as observações de Forattini et al. (1993b, 1995b) e Guimarães et al. (2000b), que destacam a importância desse culicíneo em amostragens próximas ao domicílio, pois freqüentemente vem sendo incriminado na transmissão do patógeno causador da encefalite de São Luís (ESL).

Tr. simile e Sa. chloropterus, apesar de confirmarem as expectativas pelo ambiente de mata, estações A e B, foram também capturados próximos ao convívio humano na estação $C$ (Figura 1 e Tabela 2).

A presença do Sa. chloropterus e Hg. leucocelaenus em amostragens nas proximidades dos domicílios na estação C (Tabela 2) deve ser considerada com atenção, pois ambas são incriminadas como vetoras do vírus causador da febre amarela em sua forma silvestre.

\section{Agradecimentos}

Ao Instituto Brasileiro do Meio Ambiente na pessoa do Diretor do Parque Nacional do Iguaçu, pelas facilidades concedidas para realização dos estudos. À Professora Carla Gentile, pelo auxílio na análise estatística dos dados. Aos colegas do Laboratório de Diptera do Instituto Oswaldo Cruz, pela colaboração nos trabalhos de campo. Trabalho realizado com auxílio do Conselho Nacional de Desenvolvimento Científico e Tecnológico, processo 400227/94-0. 


\section{Referências}

FORATTINI, O. P.; GOMES, A. C.; GALATI, E. A. B.; RABELO, E. X. \& IVERSSON, L. B., 1978a. Estudos ecológicos sobre mosquitos Culicidae no Sistema Serra do Mar, Brasil. 1 - Observações no ambiente extradomiciliar. Revista de Saúde Pública, 12: 297-325.

FORATTINI, O. P.; GOMES, A. C.; GALATI, E. A. B.; RABELO, E. X. \& IVERSSON, L. B., 1978b. Estudos ecológicos sobre mosquitos Culicidae no Sistema Serra do Mar, Brasil. 2 - Observações no ambiente domiciliar. Revista de Saúde Pública, 12:476496.

FORATTINI, O. P.; GOMES, A. C.; NATAL, D. \& SANTOS, J. L. F., 1986a. Observações sobre atividade de mosquitos Culicidae em mata primitiva da encosta no Vale da Ribeira, São Paulo, Brasil. Revista de Saúde Pública, 20:1-20.

FORATTINI, O. P.; GOMES, A. C.; NATAL, D. \& SANTOS, J. L. F., 1986b. Observações sobre atividade de mosquitos Culicidae em mata primitiva da planície e perfis epidemiológicos de vários ambientes no Vale da Ribeira, São Paulo, Brasil. Revista de Saúde Pública, 20:178-203.

FORATTINI, O. P.; GOMES, A. C.; SANTOS, J. L. F.; GALATI, E. A. B.; RABELO, E. X. \& NATAL, D., 1981. Observações sobre a atividade de mosquitos $\mathrm{Cu}$ licidae, em mata residual do Vale da Ribeira, São Paulo, Brasil. Revista de Saúde Pública, 15:557586.

FORATTINI, O. P.; KAKITANI, I.; MASSAD, E. \& GOMES, A. C., 1993a. Studies on mosquitos (Diptera: Culicidae) and anthropic and environment. 1 Parity of blood seeking Anopheles (Kerteszia) in Southeastern, Brazil. Revista de Saúde Pública, 27:1-8.

FORATTINI, O. P.; KAKITANI, I.; MASSAD, E. \& MARUCCI, D., 1993b. Studies on mosquitos (Diptera: Culicidae) and anthropic and anvironment. 4 Survey of resting adult and synanthropic behaviour in Southeastern, Brazil. Revista de Saúde Pública, 27:398-411.

FORATTINI, O. P.; KAKITANI, I.; MASSAD, E. \& MARUCCI, D, 1995a. Studies on mosquitoes (Diptera: Culicidae) and anthropic environment. 9 Synanthropy and epidemiological vector role of Aedes scapularis in Southeastern Brazil. Revista de Saúde Pública, 29:199-207.

FORATTINI, O. P.; KAKITANI, I.; MASSAD, E. \& MARUCCI, D, 1995b. Studies on mosquitoes (Diptera: Culicidae) and anthropic environment. $10-$ Survey of adult behaviour of Culex nigripalpus and other species of Culex (Culex) in Southeastern Brazil. Revista de Saúde Pública, 29:271-278.

GUIMARÃES, A. E. \& ARLÉ, M., 1984. Mosquitos no Parque Nacional da Serra dos Órgãos, Estado do Rio de Janeiro, Brasil. I - Distribuição estacional. Memórias do Instituto Oswaldo Cruz, 79:309-323.

GUIMARÃES, A. E.; GENTILE, C.; LOPES, C. M. \& MELLO, R. P., 2000a. Ecology of mosquitoes (Diptera: Culicidae) in areas of Serra do Mar State Park, State of São Paulo, Brazil. II - Habitat distribution. Memórias do Instituto Oswaldo Cruz, 95: $17-28$.
GUIMARÃES, A. E.; GENTILE, C.; LOPES, C. M. \& SANT'ANNA, A., 2001. Ecologia de mosquitos em áreas do Parque Nacional da Serra da Bocaina. II Freqüência mensal e fatores climáticos. Revista de Saúde Pública, 35:392-399.

GUIMARÃES, A. E.; GENTILE, C.; LOPES, C. M.; SANT'ANNA, A. \& JOVITA, A. M., 2000b. Ecologia de mosquitos em áreas do Parque Nacional da Serra da Bocaina. I - Distribuição por habitat. Revista de Saúde Pública, 34:243-250.

GUIMARÃES, A. E.; MELLO, R. P.; LOPES; C. M.; ALENCAR, J. \& GENTILE, C., 1997. Prevalência de anofelinos (Diptera: Culicidae) no crepúsculo vespertino em áreas da Usina Hidrelétrica de Itaipu, no município de Guairá, estado do Paraná, Brasil. Memórias do Instituto Oswaldo Cruz, 92 745-754.

GUIMARÃES, A. E.; MELLO, R. P.; LOPES, C. M. \& GENTILE, C., 2000c. Ecology of mosquitoes (Diptera: Culicidae) in areas of Serra do Mar State Park, State of São Paulo, Brazil. I - Monthly frequency and climatic factors. Memórias do Instituto Oswaldo Cruz, 95:1-16.

GUIMARÃES, A. E.; MOTTA, M.; ARLÉ, M.; MACHADO, R. N. M., \& GONÇALVES, L. D., 1989. Bionomia de mosquitos (Diptera-Culicidae) em áreas da mata Atlântica no município de Itaguaí, Estado do Rio de Janeiro, Brasil. I - Freqüência intra, peri e extradomiciliar. Memórias do Instituto Oswaldo Cruz, 84:243-254.

HARBACH, R. E. \& PEYTON E. L., 2000. Systematics of Onirion, a new genus of Sabethini (Diptera: Culicidae) from the Neotropical Region. Bulletin of the Natural History Museum of London (Entomology), 69:115-169.

JUDD, D. D., 1996. Review of the systematics and phylogenetic relationships of the Sabethini (Diptera: Culicidae). Systematic Entomology, 21:129-150.

JUDD, D. D., 1998. Review a bromeliad-ovipositing lineage in Wyeomyia and the resurrection of Hystatomyia. Annals of the Entomological Society of America, 91:572-589.

LOPES, C. M.; GUIMARÃES, A. E.; MELLO, R. P. \& ALENCAR, J., 1999. Ecologia de mosquitos (Diptera: Culicidae) em áreas da Reserva Biológica do Tingua, Município de Nova Iguaçu, Estado do Rio de Janeiro, Brasil. I - Diversidade faunística. Entomología y Vectores, 6:563-576.

REINERT, J. F., 1975. Mosquito generic and subgeneric abbreviations (Diptera: Culicidae). Mosquito Systematics, 7:105-110.

REINERT, J. F., 2000. New classification for the composite genus Aedes (Diptera: Culicidae: Aedini), elevation of subgenus Ochlerotatus to generic rank, reclassification of the other subgenera, and notes on certain subgenera and species. Journal of the American Mosquito Control Association, 16:175-188.

Recebido em 18 de setembro de 2002

Versão final reapresentada em 8 de janeiro de 2003 Aprovado em 9 de abril de 2003 